\title{
Temporally Controlled Modulation of Antihydrogen Production and the Temperature Scaling of Antiproton-Positron Recombination
}

\author{
M. C. Fujiwara, ${ }^{1,2, *}$ M. Amoretti, ${ }^{3}$ C. Amsler, ${ }^{4}$ G. Bonomi, ${ }^{5,6}$ A. Bouchta, ${ }^{7}$ P. D. Bowe, ${ }^{8}$ C. Canali, ${ }^{3,9}$ C. Carraro, ${ }^{3,9}$
} C. L. Cesar, ${ }^{10}$ M. Charlton, ${ }^{11}$ M. Doser, ${ }^{7}$ A. Fontana,,${ }^{6,12}$ R. Funakoshi, ${ }^{13}$ P. Genova, ${ }^{6,12}$ J. S. Hangst ${ }^{8}$ R. S. Hayano, ${ }^{13}$ L. V. Jørgensen, ${ }^{11}$ A. Kellerbauer, ${ }^{7}$ V. Lagomarsino, ${ }^{3,9}$ R. Landua, ${ }^{7}$ E. Lodi-Rizzini, ${ }^{14,15}$ M. Macri, ${ }^{3}$ N. Madsen, ${ }^{8,11}$ G. Manuzio, ${ }^{3,9}$ D. Mitchard, ${ }^{11}$ P. Montagna, ${ }^{6,12}$ H. Pruys, ${ }^{4}$ C. Regenfus, ${ }^{4}$ A. Rotondi,${ }^{6,12}$ G. Testera, ${ }^{3}$ A. Variola, ${ }^{3}$ L. Venturelli, ${ }^{14,15}$ D. P. van der Werf, ${ }^{11}$ Y. Yamazaki, ${ }^{2}$ and N. Zurlo ${ }^{14,15}$

(ATHENA Collaboration)

\author{
${ }^{1}$ TRIUMF, 4004 Wesbrook Mall, Vancouver, British Columbia, V6T 2A3, Canada \\ ${ }^{2}$ Atomic Physics Laboratory, RIKEN, Saitama 351-0198, Japan \\ ${ }^{3}$ Istituto Nazionale di Fisica Nucleare, Sezione di Genova, I-16146 Genova, Italy \\ ${ }^{4}$ Physics Institute, Zurich University, CH-8057 Zurich, Switzerland \\ ${ }^{5}$ Department of Mechanical and Industrial Engineering, University of Brescia, I-25123 Brescia, Italy \\ ${ }^{6}$ Istituto Nazionale di Fisica Nucleare, Sezione di Pavia, I-27100 Pavia, Italy \\ ${ }^{7}$ Physics Department, CERN, 1121 Geneva 23, Switzerland \\ ${ }^{8}$ Department of Physics and Astronomy, University of Aarhus, DK-8000 Aarhus C, Denmark \\ ${ }^{9}$ Dipartimento di Fisica di Genova, I-16146 Genova, Italy \\ ${ }^{10}$ Instituto de Fisica, Universidade Federal do Rio de Janeiro, Rio de Janeiro 21945-970, Brazil \\ ${ }^{11}$ Department of Physics, Swansea University, Swansea SA2 8PP, United Kingdom \\ ${ }^{12}$ Dipartimento di Fisica Nucleare e Teorica, Università di Pavia, I-27100 Pavia, Italy \\ ${ }^{13}$ Department of Physics, University of Tokyo, Tokyo 113-0033, Japan \\ ${ }^{14}$ Istituto Nazionale di Fisica Nucleare, Gruppo Collegato di Brescia, I-25123 Brescia, Italy \\ ${ }^{15}$ Dipartimento di Chimica e Fisica per l'Ingegneria e per i Materiali, I-25123 Brescia, Italy
}

(Received 2 October 2007; published 30 July 2008)

\begin{abstract}
We demonstrate temporally controlled modulation of cold antihydrogen production by periodic RF heating of a positron plasma during antiproton-positron mixing in a Penning trap. Our observations have established a pulsed source of atomic antimatter, with a rise time of about $1 \mathrm{~s}$, and a pulse length ranging from 3 to $100 \mathrm{~s}$. Time-sensitive antihydrogen detection and positron plasma diagnostics, both capabilities of the ATHENA apparatus, allowed detailed studies of the pulsing behavior, which in turn gave information on the dependence of the antihydrogen production process on the positron temperature $T$. Our data are consistent with power law scaling $T^{-1.1 \pm 0.5}$ for the production rate in the high temperature regime from $\sim 100 \mathrm{meV}$ up to $1.5 \mathrm{eV}$. This is not in accord with the behavior accepted for conventional three-body recombination.
\end{abstract}

Antihydrogen $(\overline{\mathrm{H}})$, a bound state of an antiproton $(\bar{p})$ and a positron $\left(e^{+}\right)$, is the simplest form of atomic antimatter, and as such is an excellent system for probing the fundamental symmetries between matter and antimatter. Following the production of slow antihydrogen by the ATHENA [1] and ATRAP [2] experiments, a new field of cold antiatom studies is emerging.

We have shown previously $[1,3]$ that $\overline{\mathrm{H}}$ can be produced continuously for up to $180 \mathrm{~s}$ by mixing cold plasmas of $\bar{p}$ and $e^{+}$. In this letter, we demonstrate temporally controlled production of $\overline{\mathrm{H}}$ by applying a modulated RF field to heat the $e^{+}$plasma. Our observations establish a pulsed source of cold $\overline{\mathrm{H}}$ atoms, with a rise time of about $1 \mathrm{~s}$ and pulse lengths ranging from $3 \mathrm{~s}$ to $100 \mathrm{~s}$. We note that temporal control of recombination via modulation of collision energies has previously been used to elucidate aspects of recombination physics [4].
We also report detailed studies of the temperature dependence of $\overline{\mathrm{H}}$ formation, utilizing the pulsed $\overline{\mathrm{H}}$ signal. Optimizing $\bar{p}-e^{+}$recombination is important for realization of the ambitious goal of $\overline{\mathrm{H}}$ trapping [5], but a thorough quantitative understanding of the process has yet to emerge, despite on-going theoretical efforts: see, e.g., [6-12]. We have developed a novel method of monitoring $\overline{\mathrm{H}}$ production during the synchrotron cooling of the $e^{+}$ plasma. Our data probe the previously unexplored plasma temperature range up to $1.5 \mathrm{eV}$, and suggest power law scaling of antihydrogen formation that deviates substantially from that assumed for conventional three-body recombination.

The ATHENA antihydrogen apparatus [13], located at the Antiproton Decelerator (AD) facility at CERN, consisted of several modular components, including $\bar{p}$ and mixing traps (both held at $15 \mathrm{~K}$ in a $3 \mathrm{~T}$ solenoid field), 
the $e^{+}$accumulator [14], a Si vertex detector [15,16], external detectors [17] and the associated control systems.

A few times $10^{7} \bar{p}$, obtained at $5.3 \mathrm{MeV}$ from the $\mathrm{AD}$, are slowed by degrader foils to $<5 \mathrm{keV}$, and about $10^{4}$ are captured in the $\bar{p}$ trap. After being sympathetically cooled with preloaded cold electrons [18], the $\bar{p}$ are moved to the mixing trap. Positrons are accumulated in a Surko-type nitrogen buffer gas trap [19] and transferred using a pulsed electric field into the mixing region [14]. Typically $10^{4} \bar{p}$ and around $5 \times 10^{7} e^{+}$are mixed by injecting the former into the $e^{+}$plasma in a nested potential well configuration [20]. Our $e^{+}$plasma had a typical density of a few $\times 10^{8} \mathrm{~cm}^{-3}$, and a length of $3 \mathrm{~cm}$. When an $\overline{\mathrm{H}}$ atom is formed, it may escape the electromagnetic confinement of the Penning trap and annihilate on collision with the trap wall. Such events are detected by a position sensitive imaging detector [15]; the positions of $\bar{p}$ annihilations (vertices) are determined by tracking the trajectories of the charged products (mostly pions) using the Si vertex detector [16], while $511 \mathrm{keV} \gamma$ rays from $e^{+}$annihilations are detected by segmented CsI crystals. The detection system allows time-dependent determination of $\overline{\mathrm{H}}$ production [3]. It is this ability, coupled with the real-time plasma temperature control and diagnosis techniques developed in [21], that enabled the detailed studies reported here. Note that we detect here only $\overline{\mathrm{H}}$ atoms that survive ionization from the ambient electric fields to reach the trap walls.

In our previous studies [1,3,22], $\overline{\mathrm{H}}$ formation could be suppressed by continuously heating the $e^{+}$plasma with RF tuned to the dipole plasma modes [21]. The $e^{+}$temperature could also be modulated by applying periodic RF, with the plasma temperature returning rapidly (after $\sim 1 \mathrm{~s}$ ) to the base temperature when the heating was turned off [21]. In Fig. 1, we demonstrate that $\overline{\mathrm{H}}$ formation can be modulated by applying such periodic heating to the $e^{+}$plasma.

Figure 1(a) displays the time and axial position distributions of $\bar{p}$ annihilation vertices in which the RF was applied with a square wave modulation with a $15 \mathrm{~s}$ off $-15 \mathrm{~s}$ on cycle. In Fig. 1(b) the RF modulation is applied with the opposite phase. In both cases, the annihilation events are dramatically suppressed, within a time $\$ 100 \mathrm{~ms}$ after the $\mathrm{RF}$ was turned on. The annihilations recovered within a few seconds once the RF was turned off, as the $e^{+}$temperature return to base (see below). The RF-off vertex position distributions (see Fig. 1, lower panel) are consistent with $\overline{\mathrm{H}}$ production [3,23], while those for RF-on suggest annihilations dominantly on residual gas, or possibly trapped ions [24]. The accompanying angular distribution of $511 \mathrm{keV} \gamma$ rays gave a well-established signature for simultaneous annihilations of $\bar{p}$ and $e^{+}[1,3]$. We have also demonstrated $\overline{\mathrm{H}}$ production with modulation periods ranging from 3 to $100 \mathrm{~s}$.

In order to understand the temporal behavior of the $\overline{\mathrm{H}}$ modulation, we consider a simple model as follows: turning on the RF heats the $e^{+}$plasma by $\Delta T$, immediately suppressing $\overline{\mathrm{H}}$ production. When the $\mathrm{RF}$ is turned off, the $e^{+}$plasma self-cools via emission of synchrotron radiation with a time constant $\tau_{e} \sim 0.5 \mathrm{~s}$ to the equilibrium temperature $T_{0}$, with $\overline{\mathrm{H}}$ formation recovering with a similar time constant. If the temperature evolution of the $e^{+}$

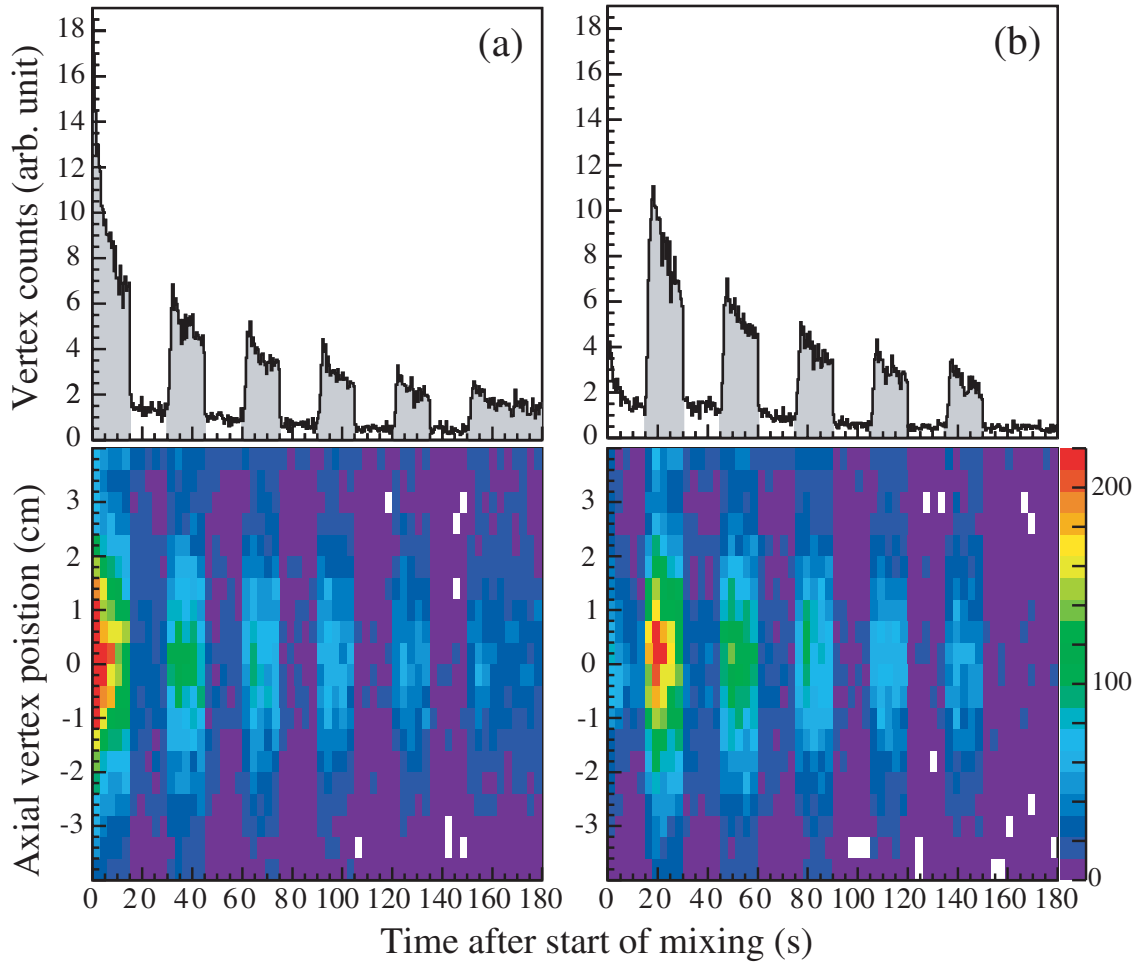

FIG. 1 (color). Spatial and temporal distributions of $\bar{p}$ annihilations during the modulated $\mathrm{RF}$ heating of the $e^{+}$ plasma, illustrating modulated $\overline{\mathrm{H}}$ production. (a) Heat on-off, and (b) off-on cycles. In the upper panels the heat off period is shaded. 


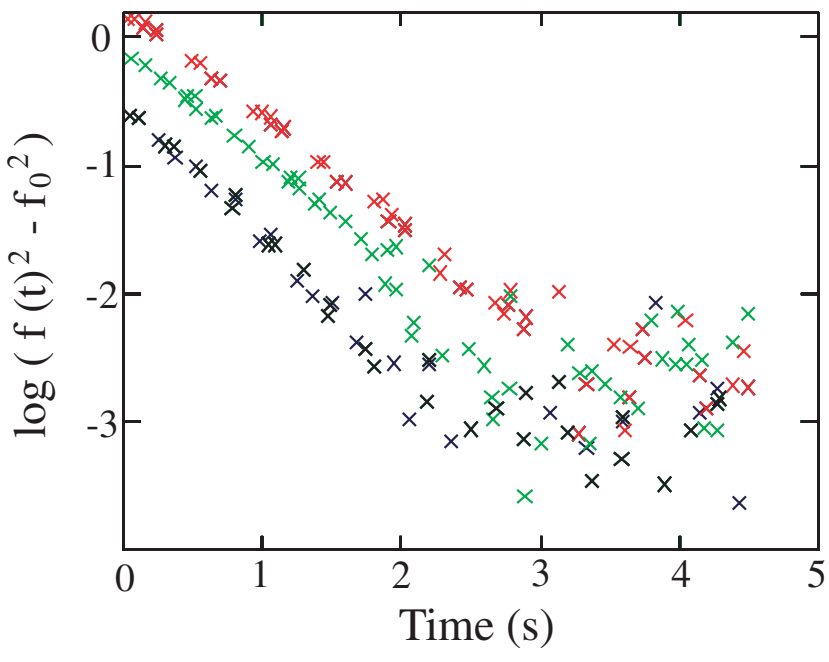

FIG. 2 (color). Measurements of $e^{+}$plasma cooling time. Plotted are $f(t)^{2}-f_{0}^{2}$ (proportional to $\Delta T$ ) versus time after $\mathrm{RF}$ removal. RF amplitudes of $25 \mathrm{mV}$ (red), $13 \mathrm{mV}$ (green), and $5 \mathrm{mV}$ (black) were applied.

plasma is known, the temperature dependence of $\overline{\mathrm{H}}$ formation can be deduced, which in turn provides information on $\overline{\mathrm{H}}$ formation dynamics in a nested trap.

We quantitatively test this model, focusing on the onset of antihydrogen production upon turning the heating off. The $e^{+}$cooling time $\tau_{e}$ at $B=3 \mathrm{~T}$ can be calculated from [25]: $\tau_{e}=0.43 \mathrm{~s}$. Experimental measurements, however, are reported to give higher values than this estimate $[25,26]$. Given the importance of this parameter in our later fits, we determine this cooling rate via analysis of the quadrupole plasma modes frequency $f$ in offline $\left(e^{+}\right.$-only) measurements, based on the method reported in [21]. The $e^{+}$are heated by applying modulated RF with a $5 \mathrm{~s}$ on $-5 \mathrm{~s}$ off cycle. According to the cold fluid model $[27,28], \Delta T$ is proportional to $f^{2}-f_{0}^{2}$ where $f_{0}$ is the equilibrium value. The evolution of the modes frequency after RF removal, $f(t)$, can be fitted to: $f(t)^{2}=$ $\kappa \Delta T \exp \left(-t / \tau_{e}\right)+f_{0}^{2}$, to extract $\tau_{e}$ and $f_{0}$. The constant $\kappa$ is uncalibrated for these measurements, but is not used for the determination of $\tau_{e}$ ( $\kappa$ will be calibrated for the $\Delta T$ measurements below). Figure 2 illustrates the exponential nature of the plasma temperature evolution, where $f^{2}(t)-$ $f_{0}^{2}$ is plotted for 3 different initial heating amplitudes. From the data, we obtain $\tau_{e}$ of $0.42 \mathrm{~s}$ (for an RF amplitude of $5 \mathrm{mV}), 0.50 \mathrm{~s}(13 \mathrm{mV})$, and $0.52 \mathrm{~s}(25 \mathrm{mV})$, giving an average of $\tau_{e}=0.48 \pm 0.05 \mathrm{~s}$. A possible systematic shift of $\tau_{e}$ with the heating amplitude is included in our uncertainty.

We now present a series of $\overline{\mathrm{H}}$ modulation data, with an RF modulation cycle of $5 \mathrm{~s}$ off $-3 \mathrm{~s}$ on. Seven periods of the on-to-off transition occurring during the $70 \mathrm{~s}$ mixing time were summed over typically 10 mixing cycles for each initial temperature, such that about 70 transitions per data set were used for the analysis. We consider here the anni-
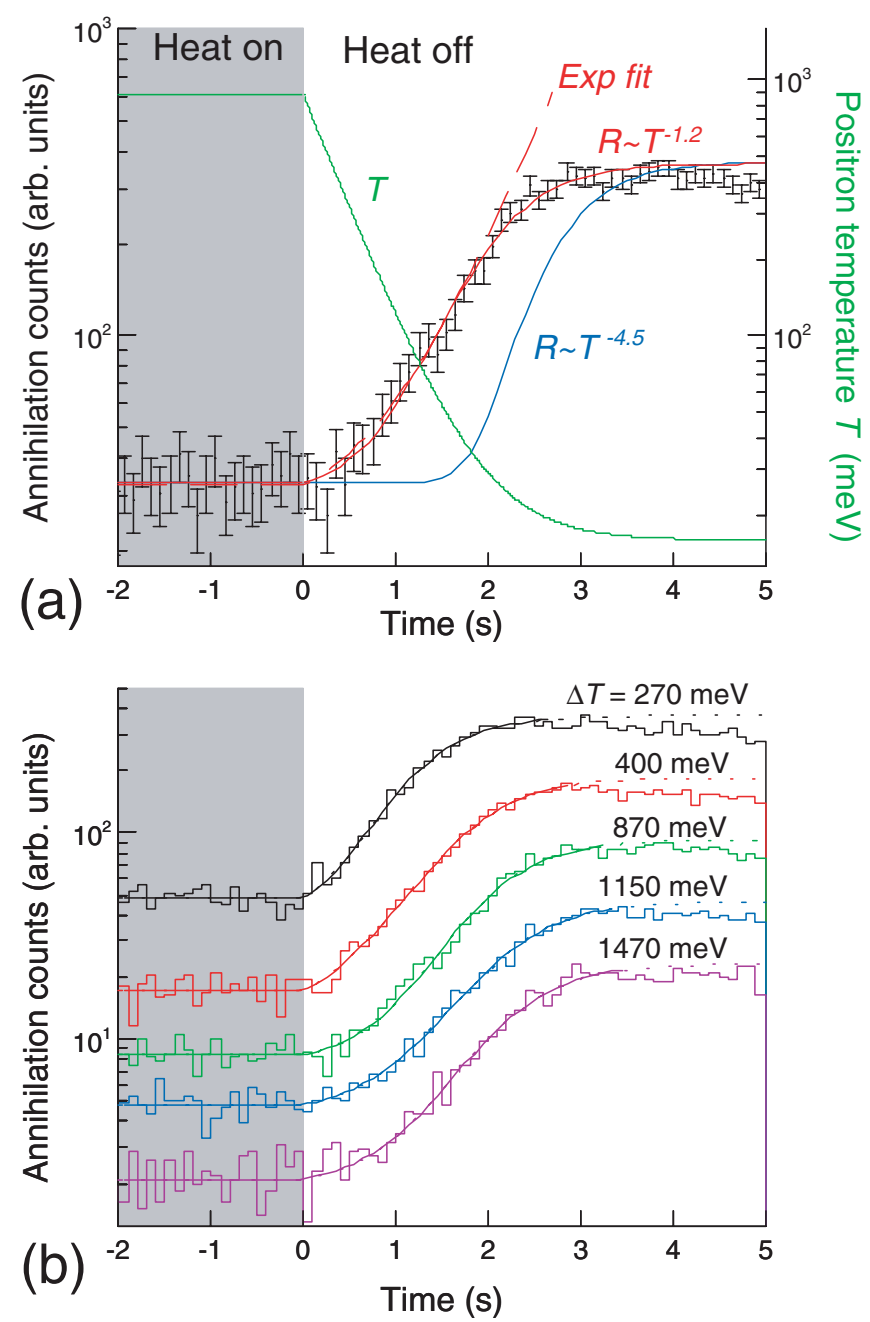

FIG. 3 (color). Onset of $\overline{\mathrm{H}}$ production upon heat off at $t=0$. (a) The data with $\Delta T=870 \mathrm{meV}$ (error bars). Green curve: the $e^{+}$temperature evolution with $\tau_{e}=0.48 \mathrm{~s}$. Red curve: a representative fit to Eq. (1), giving $R_{\overline{\mathrm{H}}} \propto T^{-1.2}$. Dashed red curve: exponential fit. Blue: the case with $R_{\overline{\mathrm{H}}} \propto T^{-4.5}$. (b) The data and representative fits for different $\Delta T$. Fit time windows (indicated by solid curves) were set with $T_{\text {low }}=1 \mathrm{meV}$ (see text). The data sets (which are vertically displaced for clarity) were taken with between $0.7-1.6 \times 10^{5} \bar{p}$.

hilation rate from the detector trigger signal, rather than the reconstructed vertex rate. The former has negligible dead time compared to the latter (at the cost of having no position information), and hence can provide more accurate time information. In Fig. 3 annihilation time distributions are shown for (a) $\Delta T=870 \mathrm{meV}$ (error bars), and (b) $270,400,870,1150$, and $1470 \mathrm{meV}$ (histograms). $\Delta T$ is obtained from the modes measurements [21], with $\kappa$ now calibrated using the resonance line shape to give the absolute temperature increase.

In order to fit these data, a temperature power scaling of the production rate, i.e. $R_{\overline{\mathrm{H}}}(T) \propto T^{-P}$, has been assumed, where $T$ is the $e^{+}$temperature. Assuming that the disap- 
pearance of the $\bar{p}$ is dominated by $\overline{\mathrm{H}}$ production over the time scale of interest ( $<$ a few seconds), the observed $\bar{p}$ annihilation rate $\lambda(t)$ can be written as

$$
\lambda(t)=A\left[\Delta T \exp \left(-\frac{t}{\tau_{e}}\right)+T_{0}\right]^{-P}+B_{k},
$$

for $t>0$, and $\lambda(t)=A\left[\Delta T+T_{0}\right]^{-P}+B_{k}$ for $t<0 . T_{0}$ (base temperature), $A$ (normalization) and $B_{k}$ (background), as well as $P$, are the fit parameters. In this model, in the limit of $T_{0} \rightarrow 0$ and no background, the slope of the onset of $\overline{\mathrm{H}}$ production in a logarithmic plot represents the power scaling $P$. Our data show indications of such scaling, especially at intermediate time where the effects of $T_{0}$ and $B_{k}$ are small. Increasing $\Delta T$ results in delayed onset of $\overline{\mathrm{H}}$ production as expected, since it takes longer for the $e^{+}$ plasma to cool down.

Representative fits are illustrated by the curves in Fig. 3 . The red curve in (a) is a fit to the $\Delta T=870 \mathrm{meV}$ data, resulting in the scaling $P=1.2 \pm 0.2$, and an effective base temperature of $T_{0}=180 \pm 50 \mathrm{~K}$, where the errors are statistical only. The green curve shows the $e^{+}$temperature evolution, where $T_{0}$ is taken from the fit above. The blue curve is the case where the conventional three-body recombination scaling $P=4.5[6]$ is assumed. Here the onset of the $\overline{\mathrm{H}}$ production would be much delayed, a scenario which is clearly incompatible with the data. Similar fits to the data with different $\Delta T$ are given in curves in Fig. 3(b). The fit time windows (indicated with solid curves) were taken to be $-2<t<\left(\tau_{e} \ln \left(\Delta T / T_{\text {low }}\right)\right) \mathrm{s}$, where the effective low temperature cut off $T_{\text {low }}$ is set to $1 \mathrm{meV}$ here.

We observe that the fitted base temperatures $T_{0}$ obtained are typically higher than the nominal trap temperature of $15 \mathrm{~K}$. In our model [Eq. (1)], deviation from power scaling at low temperatures as implied by [22], would be accounted for by a higher value of $T_{0}$ [29]. In order to avoid the influence of the possible nonpower scaling at low temperatures, we make alternative fits, limited to the high temperature region, assuming the form: $\lambda(t)=$ $A\left[\Delta T \exp \left(-t / \tau_{e}\right)\right]^{-P}+B_{k}$, for $t>0$. An example of such fit is depicted as Exp fit in Fig. 3(a), giving $P=0.8 \pm$ 0.2 with $T_{\text {low }}=35 \mathrm{meV}$.
Figure 4 illustrates consistency of the derived scaling parameters over the range of $\Delta T$ (a), and over the $70 \mathrm{~s}$ mixing time (b). The emphasis here is on the relative consistency among the data, as the absolute value of $P$ may shift due to the input parameters. In both figures, the standard fits and the exponential fits are compared.

Given the simplicity of our model, overall agreement with the data is very good. We have carefully investigated systematic uncertainties by performing a number of fits by varying, e.g., $\tau_{e}$, and $T_{\text {low }}$. The exponential fits tend to give slightly lower $P$ than the standard fits.

Taking into account these systematic effects, we obtain the scaling parameter of $P=1.1 \pm 0.5$, i.e., $R_{\bar{H}} \propto$ $T^{-1.1 \pm 0.5}$. The result is consistent with our earlier continuous heating measurements [22], but the present work substantially extends the measurements in the $100 \mathrm{meV}$ to $1.5 \mathrm{eV}$ range, and provides evidence for the power law behavior at these temperatures. Since the full range of the temperatures is probed during a single pulsing cycle, the present method should be less sensitive to some of the possible systematics, such as normalization errors. However, our determination of $P$ is highly correlated with $\tau_{e}$, and hence relies on the validity of the plasma model [27] used to extract it.

Because of the high rate of $\overline{\mathrm{H}}$ production [3], three-body recombination [6] is considered to be dominant in our experiment. However, our observed scaling is not consistent with the theoretical prediction of $R_{\overline{\mathrm{H}}} \propto T^{-4.5}$ [6], which pertains for thermal equilibrium in an infinite plasma [30]. Deviation from this scaling can be qualitatively explained by the finite size of the $e^{+}$plasma, for which nonequilibrium processes are important [8]. Such processes can produce $\overline{\mathrm{H}}$ with larger velocities $[10,23]$, which in turn has implications for its trapping in a magnetic trap [5]. Calculations in Ref. [8] suggest $P \sim 1.4$ between 15 and $30 \mathrm{~K}$, though extrapolation of this to the $\mathrm{eV}$ region requires caution. On the other hand, the consistency in Fig. 4(b) implies, within our experimental sensitivity, that the mechanism responsible for $\overline{\mathrm{H}}$ formation does not change over the time scale of 10 to $70 \mathrm{~s}$ in these experimental conditions. While the quantum state of the produced $\overline{\mathrm{H}}$ and its subsequent cascades is a subject of ongoing studies, a significant fraction of the produced $\overline{\mathrm{H}}$

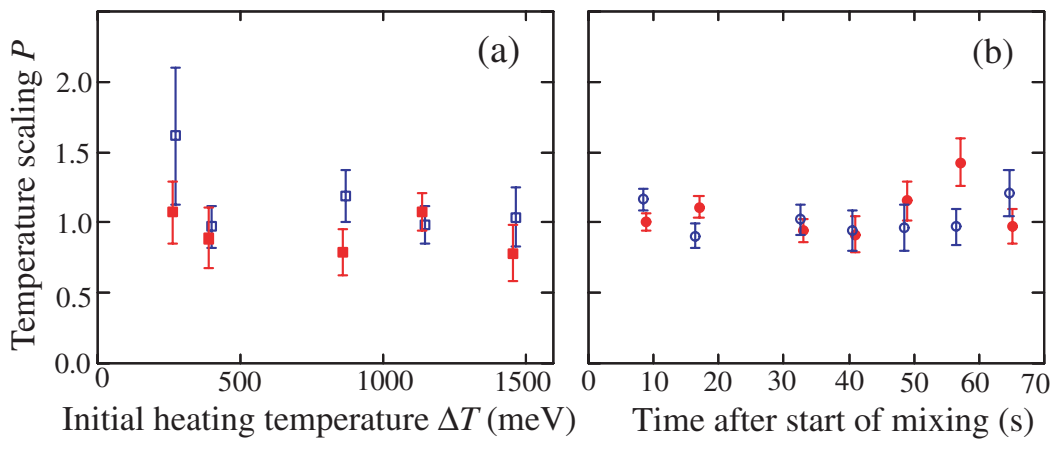

FIG. 4 (color online). Dependence of $P$ on (a) $\Delta T$ and on (b) the time after start of the mixing for the $\Delta T=$ $870 \mathrm{meV}$ data set. Open squares and circles are with the standard fits to Eq. (1), while filled ones are with the exponential fits. For the standard fits in (b), $T_{0}$ was fixed (to $150 \mathrm{~K}$ here) to achieve convergence. The plotted errors are statistical only. 
seems to reach deeper binding states in times shorter than our typical pulsing time $[7,10,11]$. Note that although the observed rise time of $\sim 1 \mathrm{~s}$ is rather modest, it could be reduced with a higher $B$ field, since $\tau_{e}$ scales scale as $1 / B^{2}$.

Finally, we remark on the possible applications of modulated $\overline{\mathrm{H}}$ production. First, the temperature scaling analysis reported here can be extended to a variety of plasma and field conditions, including nonuniform $B$ fields that are of interest for $\overline{\mathrm{H}}$ trapping [5]. Second, temporal modulation can be used as a corroborating signature for $\overline{\mathrm{H}}$ production in future experiments where standard direct detection may be difficult [31]. Third, it can be applied to low-yield and/or low-duty-cycle measurements, such as investigations of laser or microwave transitions, to give a phasesensitive signal enhancement. Furthermore, controlled modulation of $\overline{\mathrm{H}}$ production may be combined with developments in cold atom manipulation (e.g., [32]), opening up future new possibilities.

This work was supported in part by $\mathrm{CNPq}$ (Brazil), NSERC, NRC/TRIUMF (Canada), SNF (Denmark), MEXT, RIKEN (Japan), INFN (Italy), SNF (Switzerland), and the EPSRC (UK).

*Makoto.Fujiwara@triumf.ca

[1] M. Amoretti et al., Nature (London) 419, 456 (2002).

[2] G. Gabrielse et al., Phys. Rev. Lett. 89, 213401 (2002).

[3] M. Amoretti et al., Phys. Lett. B 578, 23 (2004).

[4] G. Kilgus et al., Phys. Rev. A 46, 5730 (1992); H. Gao et al., J. Phys. B 30, L499 (1997).

[5] G. Andresen et al., Phys. Rev. Lett. 98, 023402 (2007); G. Gabrielse et al. ibid. 98, 113002 (2007).

[6] M.E. Glinsky and T. M. O’Neil, Phys. Fluids B 3, 1279 (1991).
[7] E. M. Bass and D. H.E. Dubin, Phys. Plasmas 11, 1240 (2004).

[8] F. Robicheaux, Phys. Rev. A 70, 022510 (2004).

[9] B. Zygelman, J. Phys. B 38, S387 (2005).

[10] T. Pohl et al., Phys. Rev. Lett. 97, 143401 (2006).

[11] T. Topçu and F. Robicheaux, Phys. Rev. A 73, 043405 (2006).

[12] S. X. Hu, Phys. Rev. Lett. 98, 133201 (2007).

[13] M. Amoretti et al., Nucl. Instrum. Methods Phys. Res., Sect. A 518, 679 (2004).

[14] L. V. Jørgensen et al., Phys. Rev. Lett. 95, 025002 (2005).

[15] C. Regenfus, Nucl. Instrum. Methods Phys. Res., Sect. A 501, 65 (2003).

[16] M. C. Fujiwara et al., Phys. Rev. Lett. 92, 065005 (2004).

[17] M.C. Fujiwara and M. Marchesotti, Nucl. Instrum. Methods Phys. Res., Sect. A 484, 162 (2002).

[18] G. Gabrielse et al., Phys. Rev. Lett. 63, 1360 (1989).

[19] C. M. Surko et al., Phys. Rev. Lett. 62, 901 (1989).

[20] G. Gabrielse et al., Phys. Lett. A 129, 38 (1988).

[21] M. Amoretti et al., Phys. Rev. Lett. 91, 055001 (2003); Phys. Plasmas 10, 3056 (2003).

[22] M. Amoretti et al., Phys. Lett. B 583, 59 (2004).

[23] N. Madsen et al., Phys. Rev. Lett. 94, 033403 (2005).

[24] N. Zurlo et al., Phys. Rev. Lett. 97, 153401 (2006).

[25] B. R. Beck et al., Phys. Plasmas 3, 1250 (1996).

[26] A. Mohri et al., Jpn. J. Appl. Phys. 37, 664 (1998).

[27] D. H. E. Dubin, Phys. Rev. Lett. 66, 2076 (1991).

[28] M. D. Tinkle et al., Phys. Rev. Lett. 72, 352 (1994).

[29] We cannot, however, exclude from these data the possiblity of $T_{0}>15 \mathrm{~K}$, due, e.g., to some plasma heating processes, since we have no direct measurement of $T_{0}$.

[30] In fact, from the scaling alone, we cannot rule out the contribution from radiative recombination $\left(R \sim T^{-0.6}\right)$, although its predicted rate, assuming no magnetic field, is too small by at least an order of magnitude. See [22].

[31] E.g., M. C. Fujiwara, AIP Conf. Proc. 793, 111 (2005).

[32] N. Vanhaecke et al., Phys. Rev. A 75, 031402(R) (2007); E. Narevicius et al., Phys. Rev. Lett. 100, 093003 (2008). 